of gynecologic cancer patients between February and April 2020.

Methods Based on published recommendations, with local Health Direction guidelines we draft the Security Protocol to modulate the access of patients into the $\mathrm{DH}$ : to perform visits only of new patients or for clinical urgency and to convert on telemedicine the other contacts; to perform a phone/telematic pre-triage the day before the scheduled access and an 'in site' pre-triage with measurement of body temperature and administration of a survey for the self-certification of absence risk factors for COVID-19 infection; no caregivers were allowed into $\mathrm{DH}$; surgical masks and gloves were obligatory for anyone.

Results We registered 3223 accesses/contacts into our DH for intravenous/oral chemotherapy and visits. The activity was similar to that recorded in 2019 in the same two-month period (3311 accesses). Despite the high patients flow we had only two cases of confirmed COVID-19 infection and no cases among healthcare staff.

Conclusions Based on the adopted Security Protocol we have guaranteed continuity of care to all our patients and limited the spread of the COVID-19 infection.
IGCS20_1102

\section{PROGNOSTIC VALUE AND ASSOCIATION WITH VELIPARIB BENEFIT OF MODELED CA-125 ELIMINATION KINETICS (KELIM) IN PATIENTS WITH NEWLY DIAGNOSED OVARIAN CANCER: ANALYSIS FROM THE VELIA/GOG-3005 STUDY}

${ }^{1} \mathrm{~B}$ You*,${ }^{2} \mathrm{G}$ Fleming, ${ }^{3} \mathrm{M}$ Bookman, ${ }^{4} \mathrm{KN}$ Moore, ${ }^{5} \mathrm{KD}$ Steffensen, ${ }^{6} \mathrm{RL}$ Coleman. ${ }^{1} \mathrm{EMR}$ UCBL/HCL 3738, Univ Lyon, Université Claude Bernard Lyon 1, Faculté de Médecine LyonSud, GINECO, and Centre d'Investigation des Thérapeutiques en Oncologie et Hématologie de Lyon (CITOHL), Hospices Civils de Lyon (IC-HCL), France; ${ }^{2}$ AbbVie, USA; ${ }^{3}$ University of Chicago Medicine, USA; ${ }^{4}$ Kaiser Permanente Northern California, USA; ${ }^{5}$ Stephenson Cancer Center at the University of Oklahoma Health Sciences Center, USA; ${ }^{6}$ Lillebaelt University Hospital of Southern Denmark, and the University of Southern Denmark, Denmark

\subsection{6/ijgc-2020-IGCS.36}

Introduction In VELIA (Phase 3), veliparib with carboplatin/ paclitaxel (CP), followed by veliparib maintenance (veliparibthroughout) led to improved progression-free survival (PFS) vs $\mathrm{CP}$ alone (control). This exploratory analysis assessed the

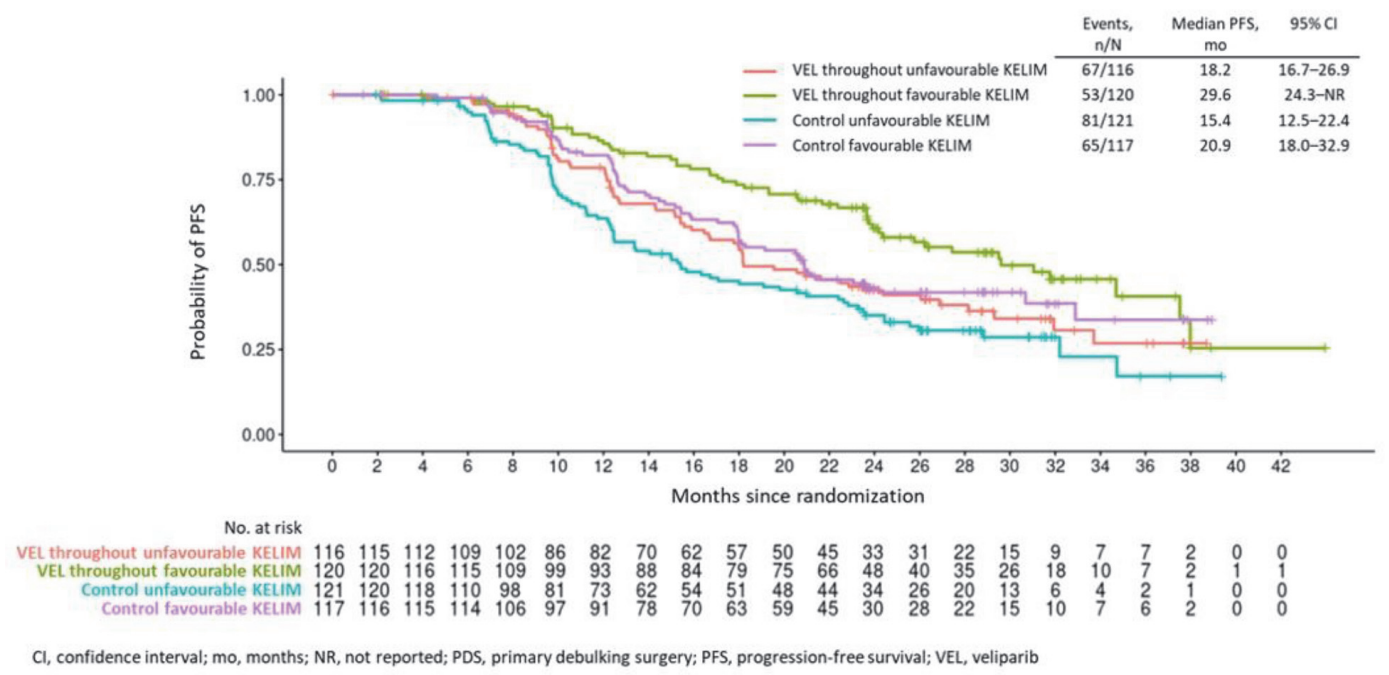

Abstract 36 Figure 1 Progression-free survival in the veliparib throughout arm and the control arm for KELIM subgroups in the PDS population

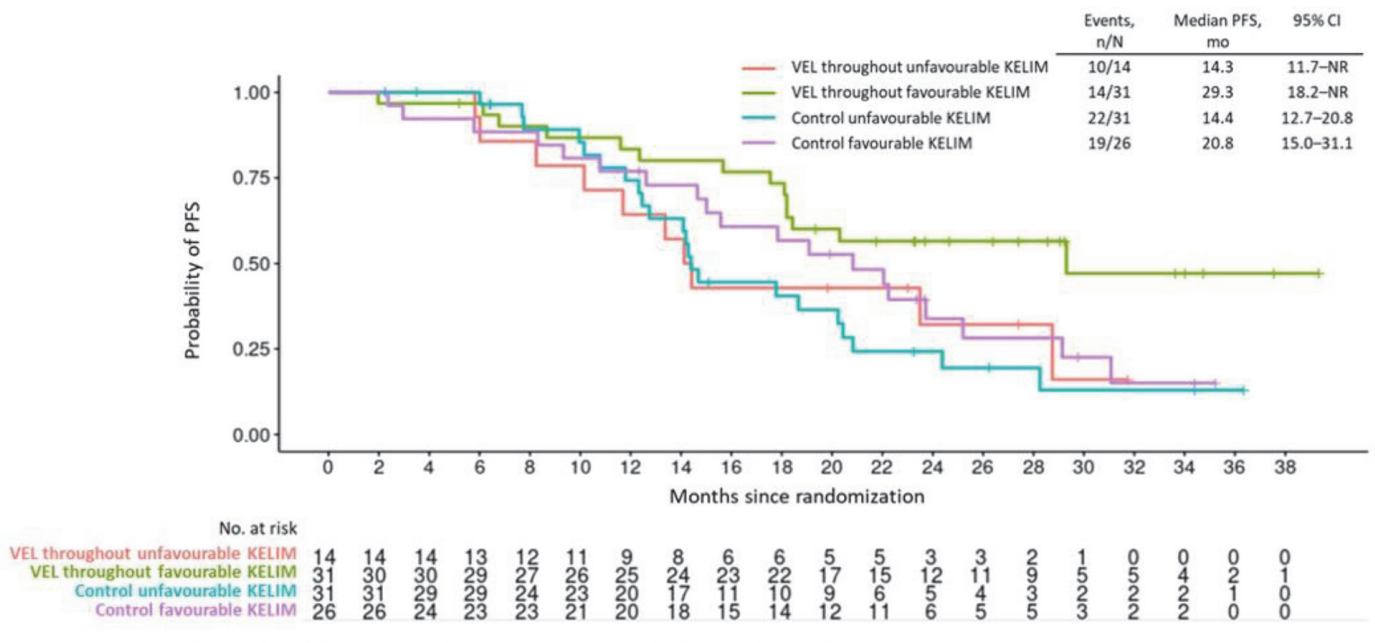

$\mathrm{Cl}$, confidence interval; mo, months; NR, not reported; IDS, interval debulking surgery; PFS, progression-free survival; VEL, veliparib

Abstract 36 Figure 2 Progression-free survival in the veliparib throughout arm and the control arm for KELIM subgroups in the IDS population 
prognostic and predictive value of the modeled CA-125 elimination rate constant, KELIM.

Methods KELIM was estimated from treatment-related pharmacodynamic modeling of CA-125 values. Median KELIM was used to define favourable ( $\geq$ median)/unfavourable (<median) KELIM groups. Patients were analyzed by surgery type: primary (PDS) or interval (IDS) debulking surgery.

Results In the IDS population $(\mathrm{N}=154)$, patients with favourable KELIM had a higher frequency of complete surgery vs unfavourable KELIM (51.9\% vs $32.4 \%)$, confirming KELIM as a chemosensitivity marker. In both PDS $(\mathrm{N}=700)$ and IDS populations, median PFS was longer with favourable KELIM vs unfavourable KELIM, demonstrating a prognostic value. In the PDS population, median PFS was longer in the veliparib-throughout arm relative to control irrespective of KELIM (29.6 vs. 20.9 and 18.2 vs 15.4 months in favourable and unfavourable KELIM groups, respectively; figure 1). In the IDS population, median PFS was longer with veliparib-throughout vs control for patients with favourable KELIM only (29.3 vs 20.8 months; figure 2).

Conclusion In VELIA, KELIM was prognostic for PFS and IDS outcomes. Current data suggest KELIM may be associated with veliparib benefit. Ongoing analyses will explore how baseline characteristics contribute to KELIM predictive/prognostic value.

\section{IGCS20_1165}

\section{QUANTITATIVE EVALUATION OF LYMPH-VASCULAR SPACE INVASION (LVSI) IN PATIENTS AFFECTED BY ENDOMETRIAL CANCER: PROGNOSTIC AND CLINICAL IMPLICATIONS}

${ }^{1} \mathrm{~S}$ Restaino*, ${ }^{1} \mathrm{~L}$ Tortorella, ${ }^{1} \mathrm{E}$ Perrone, ${ }^{1} \mathrm{G}$ Monterossi, ${ }^{2} \mathrm{~A}$ Gioè, ${ }^{2}$ I Capasso, ${ }^{2} \mathrm{E}$ La Fera, ${ }^{2} \mathrm{G}$ Dinoi, ${ }^{2} \mathrm{G}$ Scambia, ${ }^{2} \mathrm{~F}$ Fanfani, ${ }^{3} \mathrm{G}$ Zannoni, ${ }^{4} \mathrm{~V}$ Chiantera. ${ }^{1}$ Fondazione Policlinico Universitario A. Gemelli IRCCS, UOC di Ginecologia Oncologica, Dipartimento per la Tutela della Salute della Donna e della Vita Nascente, Italy; ${ }^{2}$ Università Cattolica del Sacro Cuore, Italy; ${ }^{3}$ Department of Histopathology, Università Cattolica del S. Cuore, Italy; ${ }^{4}$ Department of Gynecologic Oncology, University of Palermo, Italy

\subsection{6/ijgc-2020-IGCS.37}

Objectives Lymph-vascular space invasion (LVSI) is associated with an increased risk of recurrence. Usually, the interpretation of LVSI is just qualitative, as presence or absence. The aim of this study is to examine the quantitative analysis of LVSI.

Methods Retrospective multicentre study. It included 2300 consecutive patients who received a histologically confirmed diagnosis of endometrial cancer between January 2000 and December 2018 at the Gynaecologic Oncology Unit of two tertiary hospitals in Italy.

Results The rate of lymph node metastasis increased from the $5 \%$ in patients with no LVSI, to $15 \%$ in patients with focal LVSI and $33 \%$ in those with diffuse LVSI ( $p<$ $0.001)$. There is a progressive increase of prognostic impact of focal and diffuse LVSI on the risk of node metastasis $(\mathrm{AOR}=2.12,95 \%$ CI 1.19, 3.79 for focal LVSI and AOR $=3.67,95 \%$ CI 2.30, 5.86). Distant recurrences were more frequent in patients with diffuse LVSI compared with no LVSI (24.9\% vs $6.6 \%$ respectively, p $<0.001)$. We found that adjuvant treatments, specifically adjuvant radiations, were associated with improved OS and DFS (median DFS $=19$ months vs 97 months respectively for no adjuvant and radiation therapy, $\mathrm{p}<0.001$ ) in patients with diffuse LVSI.

Conclusion The presence of diffuse LVSI is an independent risk factor of both lymph node metastasis and distant recurrence in endometrial cancer patients. It is associated with decreased OS and DFS. Adjuvant radiation improved survival regardless of grading, histotype, and lymph nodal metastasis in women with diffuse LVSI.

\section{IGCS20_1184}

\section{A TRAINING PROGRAM TO BUILD CAPACITY FOR CERVICAL CANCER PREVENTION IN MOZAMBIQUE}

${ }^{1} \mathrm{M}$ Salcedo* ${ }^{2} \mathrm{M}$ Varon, ${ }^{2} \mathrm{M}$ Varon, ${ }^{2} \mathrm{E}$ Baker, ${ }^{2} \mathrm{E}$ Baker, ${ }^{3} \mathrm{~N}$ Osman, ${ }^{4} \mathrm{E}$ David, ${ }^{5} \mathrm{R}$ Rangeiro, ${ }^{5} \mathrm{D}$ Changule, ${ }^{6} \mathrm{~V}$ Andrade, ${ }^{7} \mathrm{CM}$ Oliveira, ${ }^{8} \mathrm{~A}$ Neves, ${ }^{9} \mathrm{~J}$ Carns, ${ }^{5} \mathrm{C}$ Lorenzoni, ${ }^{2} \mathrm{KM}$ Schmeler, ${ }^{2} \mathrm{KM}$ Schmeler. ${ }^{1}$ Obstetrics and Gynecology Department, Federal University of Health Sciences of Porto Alegre/lrmandade Santa Casa de Misericordia de Porto Alegre, Brazili ${ }^{2}$ Department of Obstetrics and Gynecology, King Chulalongkorn Memorial Hospital, Thailand; ${ }^{3}$ Universidade Eduardo Mondlane (UEM), Mozambique; ${ }^{4}$ Ministerio da Saude de Moçambique (MISAU), Mozambique; ${ }^{5}$ Hospital Central de Maputo, Mozambique; ${ }^{6} \mathrm{Hospital}$ de Câncer de Barretos, Brazil; 'Diagnóstico das Américas-DASA, Brazil; ${ }^{8}$ Hospital Geral José Macamo, Mozambique; ${ }^{9}$ Department of Bioengineering, Rice University, USA

\subsection{6/ijgc-2020-IGCS.38}

Introduction Cervical cancer is the primary cause of cancer among women in Mozambique. There is a shortage of providers trained to deliver cervical cancer screening and manage pre-invasive lesions. We describe a training program to build local capacity to deliver quality cervical cancer prevention services in Mozambique.

Methods The program includes training courses led by faculty from the United States (US) and Brazil, and consists of lectures followed by hands-on training stations to practice colposcopy, cervical biopsy, ablation and loop electrosurgical excision procedure (LEEP) using innovative training models. Participants then perform the procedures in clinic with supportive supervision from the international faculty. The courses are complemented by monthly videoconference telementoring sessions, held in Portuguese using the Project $\mathrm{ECHO}^{\circledR}$ (Extension for Community Healthcare Outcomes) model.

Results From 2016 to 2020, 10 courses were held in the cities of Maputo $(n=8)$, Beira $(n=1)$ and Nampula $(n=1)$. There were 347 participants with an average of 34 participants per course. The courses have recently transitioned from only international faculty to include Mozambican gynecologists, including two fellows from the IGCS Global Curriculum program. A total of $15 \mathrm{ECHO}$ sessions were held with $\sim 25$ participants/session, 30 patient cases discussed and 14 lectures delivered.

Conclusion This collaboration between Mozambique, Brazil and the US is building local capacity to prevent cervical cancer through training, mentoring and support of local providers. Due to the COVID-19 pandemic, the courses are being transitioned to a virtual format led by the Mozambican doctors with the international faculty joining remotely. 